\title{
Nanograting formation on metals in air with interfering femtosecond laser pulses
}

\section{$\operatorname{AUTHOR}(\mathrm{S})$ :}

Miyazaki, Kenzo; Miyaji, Godai; Inoue, Toshishige

\section{CITATION:}

Miyazaki, Kenzo ...[et al]. Nanograting formation on metals in air with interfering femtosecond laser pulses. Applied Physics Letters 2015, 107(7): 071103.

\section{ISSUE DATE:}

2015-08-17

URL:

http://hdl.handle.net/2433/201897

\section{RIGHT:}

(c) 2015 American Institute of Physics. This article may be downloaded for personal use only. Any other use requires prior permission of the author and the American Institute of Physics. 




\section{Nanograting formation on metals in air with interfering femtosecond laser pulses}

Kenzo Miyazaki, Godai Miyaji, and Toshishige Inoue

Citation: Applied Physics Letters 107, 071103 (2015); doi: 10.1063/1.4928670

View online: http://dx.doi.org/10.1063/1.4928670

View Table of Contents: http://scitation.aip.org/content/aip/journal/apl/107/7?ver=pdfcov

Published by the AIP Publishing

\section{Articles you may be interested in}

Plasmonic formation mechanism of periodic 100-nm-structures upon femtosecond laser irradiation of silicon in water

J. Appl. Phys. 116, 074902 (2014); 10.1063/1.4887808

Direct writing of continuous and discontinuous sub-wavelength periodic surface structures on single-crystalline silicon using femtosecond laser

Appl. Phys. Lett. 104, 222103 (2014); 10.1063/1.4881556

Nanograting formation through surface plasmon fields induced by femtosecond laser pulses

J. Appl. Phys. 114, 153108 (2013); 10.1063/1.4826078

Nanostructure and microripple formation on the surface of sapphire with femtosecond laser pulses

J. Appl. Phys. 111, 093518 (2012); 10.1063/1.4707951

Surface-plasmon-induced enhancement of magneto-optical Kerr effect in all-nickel subwavelength nanogratings Appl. Phys. Lett. 97, 261908 (2010); 10.1063/1.3533260

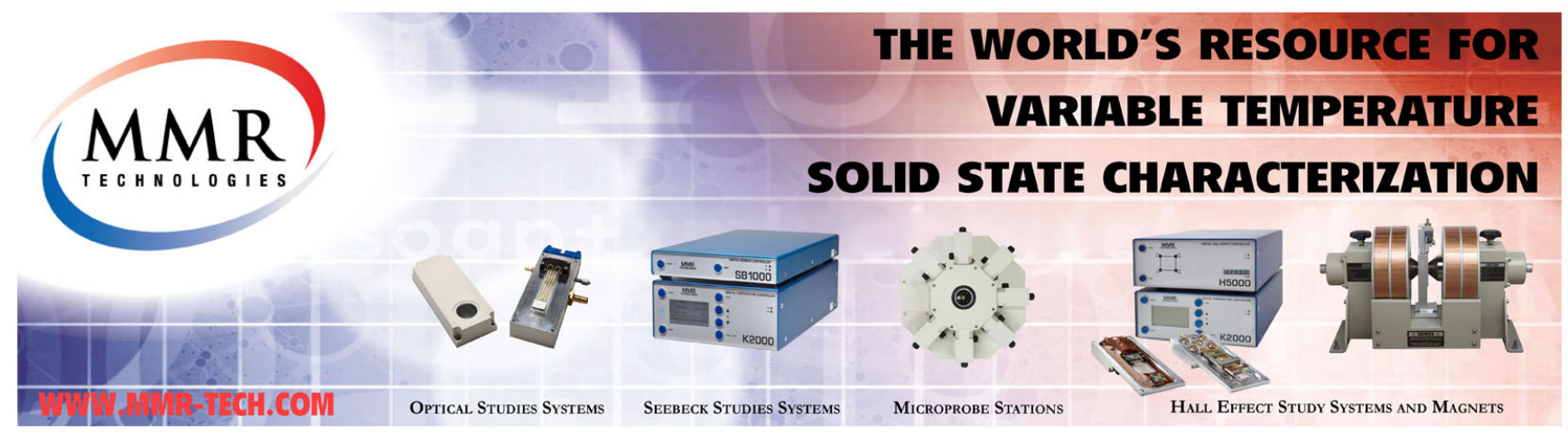




\title{
Nanograting formation on metals in air with interfering femtosecond laser pulses
}

\author{
Kenzo Miyazaki, ${ }^{1,2, a)}$ Godai Miyaji, $^{1,3, b)}$ and Toshishige Inoue ${ }^{1}$ \\ ${ }^{1}$ Laser Science Research Section, Institute of Advanced Energy, Kyoto University, Uji, Kyoto 611-0011, Japan \\ ${ }^{2}$ Center for Collaborative Research and Community Cooperation, University of Miyazaki, Miyazaki 889-2192, \\ Japan \\ ${ }^{3}$ Department of Applied Physics, Tokyo University of Agriculture and Technology, Koganei, Tokyo 184-8588, \\ Japan
}

(Received 30 June 2015; accepted 5 August 2015; published online 17 August 2015)

\begin{abstract}
It is demonstrated that a homogeneous nanograting having the groove period much smaller than the laser wavelength $(\sim 800 \mathrm{~nm})$ can be fabricated on metals in air through ablation induced by interfering femtosecond laser pulses (100 fs at a repetition rate of $10 \mathrm{~Hz}$ ). Morphological changes on stainless steel and Ti surfaces, observed with an increase in superimposed shots of the laser pulses at a low fluence, have shown that the nanograting is developed through bonding structure change at the interference fringes, plasmonic near-field ablation to create parallel grooves on the fringe, and subsequent excitation of surface plasmon polaritons to regulate the groove intervals at $1 / 3$ or $1 / 4$ of the fringe period over the whole irradiated area. Calculation for a model target having a thin oxide layer on the metal substrate reproduces well the observed groove periods and explains the mechanism for the nanograting formation. (C) 2015 AIP Publishing LLC.
\end{abstract}

[http://dx.doi.org/10.1063/1.4928670]

Self-organization process in femtosecond (fs) laser ablation of solid materials has often been observed to form a periodic nanostructure (PNS) on the target surface, where the groove period reaches a size as small as $1 / 5-1 / 10$ of the laser wavelength $\lambda .^{1-6}$ There has been a growing interest in this phenomenon, as it suggests a promising approach to nanoprocessing of materials. Extensive studies have been made to understand the mechanism responsible for the PNS formation, especially for non-metallic materials such as dielectrics and semiconductors. ${ }^{7,8}$ The experimental condition or laser parameters for nanostructuring have been identified for each target, whereas discussion on the detailed mechanism appears to still be diverse.

We have been noting the PNS formation under irradiation with superimposed multiple shots of fs laser pulses at a moderate fluence. This pump condition is crucial for the PNS formation especially in air and liquid environments, so as to avoid strong ionization of ambient materials and undesirable thermal processes. ${ }^{9,10}$ Under this condition, we have shown that a flat target surface is nanostructured through bonding structure change of the material, ${ }^{11-13}$ near-field ablation around the corrugated nanosurface, ${ }^{13-15}$ and excitation of standing surface plasmon polariton (SPP) waves. ${ }^{15-17}$ These interaction processes could explain the origin and growth of PNSs on DLC, ${ }^{15} \mathrm{Si}^{16}$ and $\mathrm{GaN},{ }^{17}$ where the theoretical calculation reproduced well the observed nanoperiod much smaller than $\sim \lambda / 2$. Based on the mechanism, we developed a two-step ablation technique to control the interaction and imprint a homogeneous nanograting in air. ${ }^{17}$

In contrast, only a few kinds of metals are known of which surfaces have been periodically nanostructured

\footnotetext{
${ }^{a)}$ Electronic addresses: k-miyazaki@iae.kyoto-u.ac.jp and kmiyazaki@wind. ocn.ne.jp

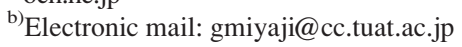

through the fs-laser ablation in air. Stainless steel (SUS) ${ }^{18-20}$ and $\mathrm{Ti}^{7,21-24}$ are the most common metals structured at the groove period smaller than $\sim \lambda / 2$, while the PNS formation was observed for $\mathrm{Cu},{ }^{25,26} \mathrm{Al}^{27}$ and $\mathrm{W}^{28}$ under the specific conditions. In the fs-laser ablation of metals, the relative lack of PNS is most likely due to the predominant formation of so-called ripples with the period size on the order of $\lambda$. The ripple has traditionally been recognized as the structure originating from the interference between the incident laser field and the surface electromagnetic waves. ${ }^{29}$ As is well known, however, this mechanism can never explain the PNS formation. The fs-laser-induced nanostructuring of metals is then far from being fully understood, and its successful control remains missing as well.

In this letter, using two-beam interference of fs laser pulses, we demonstrate that the excitation of SPPs is also responsible for the PNS formation on metals, and the interaction can be controlled to fabricate a homogeneous nanograting in air under the experimental condition of interest. The nanograting has a groove period equal to $1 / 3$ or $1 / 4$ of the interference fringe period, as the interfering fs laser pulses can excite SPPs in a single standing wave mode. Calculation for a model target has reproduced well the observed grating period to illustrate the mechanism.

The experiment was made for SUS304 and Ti targets with polished flat surfaces, using linearly polarized $800-\mathrm{nm}$, 100-fs laser pulses from a Ti:sapphire laser system operated at a repetition rate of $10 \mathrm{~Hz}$. In a preliminary experiment, we measured the single-shot ablation threshold as $F_{1}=270$ $( \pm 30)$ and $260( \pm 30) \mathrm{mJ} / \mathrm{cm}^{2}$ for the SUS and Ti targets, respectively. With superimposed multiple shots of fs laser pulses at a fluence less than $F_{1}$, periodic surface structures were spontaneously formed on these targets.

The optical setup using two beams for ablation was almost the same as that in our previous experiment. ${ }^{17}$ 


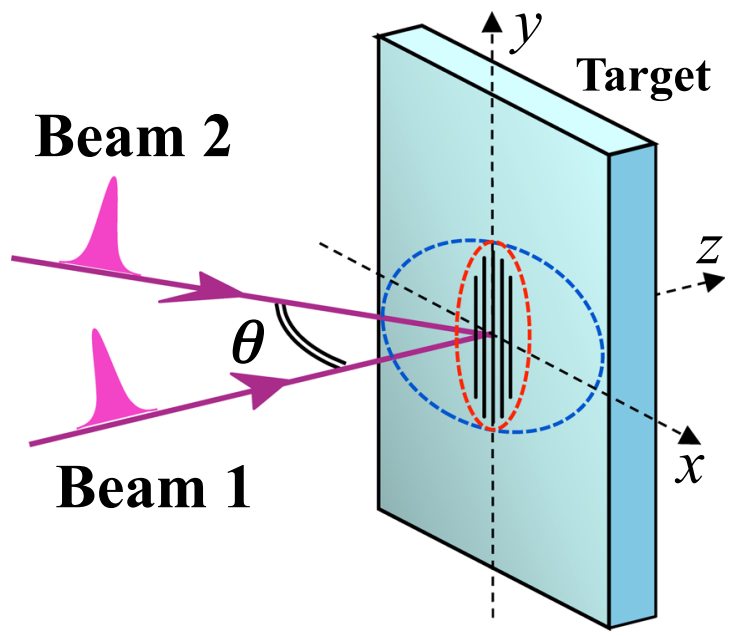

FIG. 1. Schematic of the optical configuration for pump with interfering fslaser beams, where beams 1 and 2, polarized in the $x$-z plane, are normally and obliquely incident on the target ( $x-y$ plane) at the relative angle $\theta$ to produce interference fringes in the vertical direction.

Briefly, the fs laser output was split into two beams (1 and 2) and combined again to overlap in space and time on the target surface. As schematically shown in Fig. 1, the horizontally polarized beam 1 and beam 2 are normally and obliquely incident on the target, respectively, at the relative angle $\theta$ to form an interference pattern with the fringe period $\Lambda=\lambda / \sin \theta{ }^{30}$ The beam 1 (beam 2) was focused with a 200 $\mathrm{mm}(170-\mathrm{mm})$ focal-length lens to the focal spot of $100 \mu \mathrm{m}$ $(70 \mu \mathrm{m})$ in diameter. The peak fluences $F^{(1)}$ and $F^{(2)}$ of beam 1 and 2 were independently controlled with a pair of halfwave plate and polarizer to achieve a well-defined fringe pattern at $F^{(1)}=F^{(2)}(\equiv F)$.

We observed morphological change of the target surface as a function of the superimposed shot number $N$ of interfering fs laser pulses, using a scanning electron microscope (SEM) and a scanning probe microscope (SPM). A homogeneous nanograting was formed on the surface under irradiation with $N=30-250$ at $F=40-100 \mathrm{~mJ} / \mathrm{cm}^{2}$, while a higher fluence or an excess shot number was observed to disturb the uniform grating period.

Figure 2 shows the SEM image and its Fourier spectrum observed for SUS with increasing $N$ at $F=50 \mathrm{~mJ} / \mathrm{cm}^{2}$, using the interfering beams with $\Lambda=890 \mathrm{~nm}$ at $\theta=64^{\circ}$, where the peak fluence at the fringes (bold arrows) is much smaller than $F_{1}$. The bright and dark stripes in the image correspond to the ridge and groove in the surface structure, respectively. After $N=10$, discontinuous broad line-like spots are created along the interference pattern [Fig. 2(a)]. With $N=20$, a more distinct fringe pattern is recorded [Fig. 2(b)], where one or two narrow line-like valleys are produced through ablation on the fringe. With a further increase in $N$, the narrow line-like grooves tend to be generated with the period less than $\Lambda$ on the whole area [Fig. 2(c)]. After $N=150$, the surface structure is a homogeneous nanograting with the groove period $d \sim \Lambda / 3=297 \mathrm{~nm}$, as clearly seen with the isolated peak at $3 / \Lambda$ in the spectrum [Fig. 3(d)]. This indicates that intense nanofields for ablation are periodically generated to regulate the groove intervals at $d \sim \Lambda / 3$.
The experiment using the interfering beams was also made for Ti. Figure 3 shows the SEM image and its Fourier spectrum observed with increasing $N$ at $F=50 \mathrm{~mJ} / \mathrm{cm}^{2}$. For $N=5$, broad ablation traces are created on the fringe pattern [Fig. 3(a)]. With an increase in $N$, the fringe pattern is changed into a structure with periodic nanogrooves [Fig. 3(b)], and the groove period is regulated to form a nanograting with $d \sim \Lambda / 3$ [Fig. 3(c)], similar to those shown in Fig. 2.

To see in more detail the growth of a nanograting, the experiment was made for the extended fringe period $\Lambda=1400 \mathrm{~nm}$ at $\theta=34^{\circ}$. Figure 4 shows the SEM images of the SUS surface irradiated with the different shot numbers at $F=50 \mathrm{~mJ} / \mathrm{cm}^{2}$ ((a) and (b)) and $F=100 \mathrm{~mJ} / \mathrm{cm}^{2}$ ((c) and (d)).

At $F=50 \mathrm{~mJ} / \mathrm{cm}^{2}$, the fringe pattern similar to that shown in Fig. 2(a) was produced with a few tens of shots. After $N=150$, as seen in Fig. 4(a), one or two narrow linelike ablation traces are created on a fringe. With $N=250$, additional ablation is induced to form a set of three parallel line-like grooves on the fringe [Fig. 4(b)], which consists of one at the intensity peak (thick solid arrow) and two on its both sides (thin solid arrows). There is no distinct difference in the width among the three grooves, despite the large spatial gradient of laser intensity at the fringe. These results, together with those in Figs. 2(b) and 3(a), indicate that the narrow line-like grooves are produced through the near-field

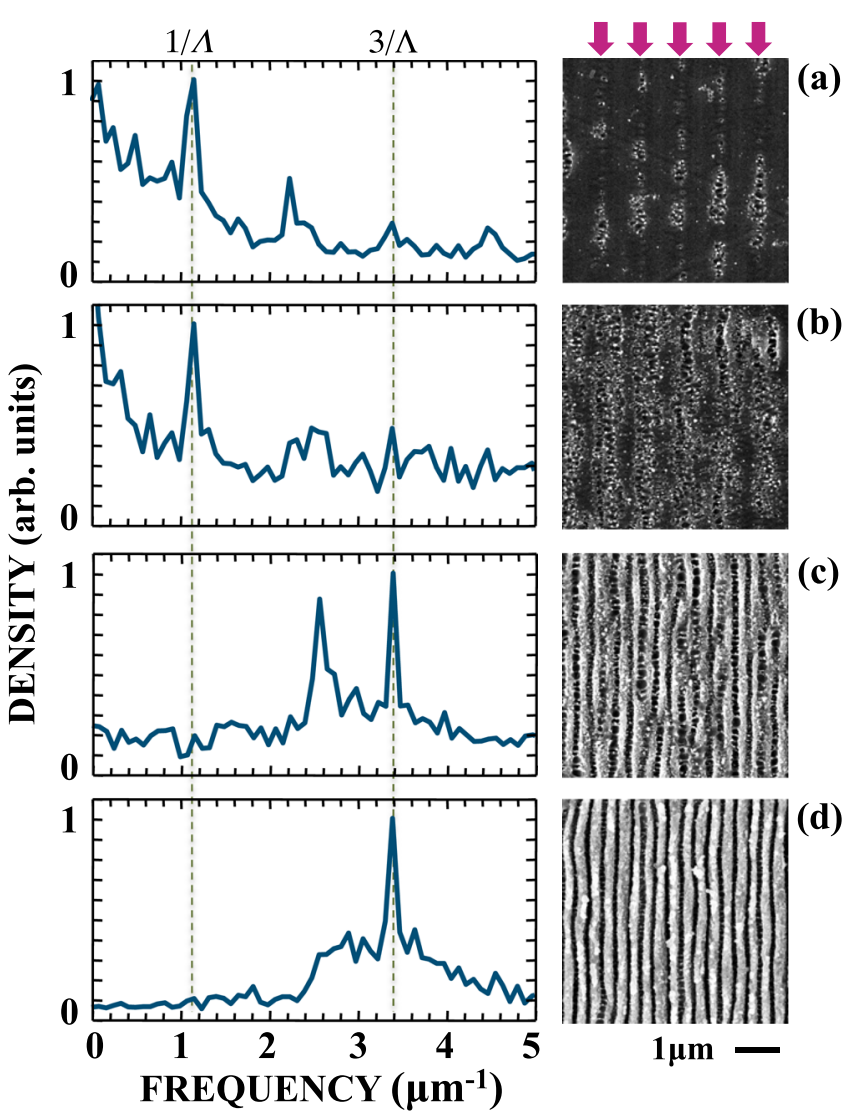

FIG. 2. SEM image and its frequency spectrum of the periodic structure on SUS surface irradiated with (a) $N=10$, (b) $N=20$, (c) $N=50$, and (d) $N=150$ at $F=50 \mathrm{~mJ} / \mathrm{cm}^{2}$ for $\Lambda=890 \mathrm{~nm}$. The laser polarization is horizontal on the images, and the arrows above the image represent the fringe positions for reference. The vertical dashed lines in the spectra indicate the frequencies at $1 / \Lambda$ and $3 / \Lambda$. 


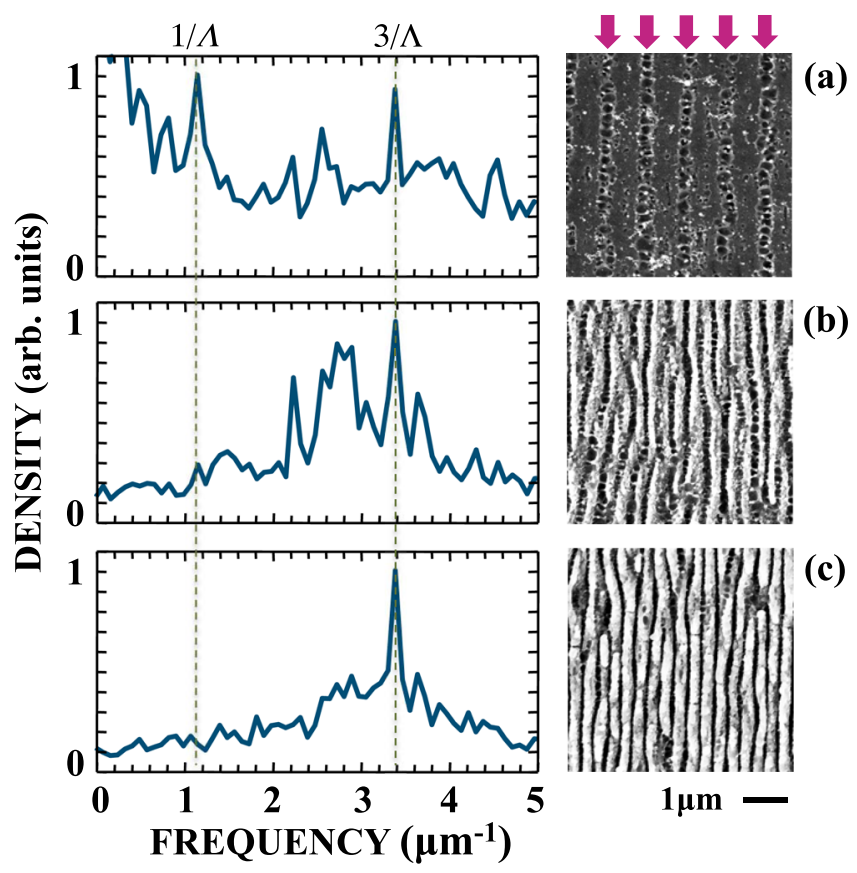

FIG. 3. SEM image and its frequency spectrum of the periodic structure on Ti surface irradiated with (a) $N=5$, (b) $N=30$, and (c) $N=100$ at $F=50$ $\mathrm{mJ} / \mathrm{cm}^{2}$ for $\Lambda=890 \mathrm{~nm}$. The laser polarization is horizontal on the images, and the arrows above the image represent the fringe positions for reference. The vertical dashed lines in the spectra indicate the frequencies at $1 / \Lambda$ and $3 / \Lambda$.

ablation around high curvatures on the fringe, as the fringe surface is inevitably swelled through the bonding structure change. ${ }^{13-15}$ Note that the spacing between the adjacent grooves corresponds to the size of near-fields. ${ }^{31}$ With a further increase in $N$, the line-like grooves developed to form a nanograting with $d \sim \Lambda / 4=350 \mathrm{~nm}$.

At $F=100 \mathrm{~mJ} / \mathrm{cm}^{2}$, the visible interference pattern was created with only a few shots of fs pulses, due to the fluence higher than $F_{1}$ at the fringes. After $N=50$, the surface image [Fig. 4(c)] represents a set of three line-like grooves on the fringe, similar to that in Fig. 4(b). Meanwhile, additional shallow ablation traces (thin white arrows) are produced on the midway between two adjacent fringes where the laser intensity is minimized in the interference pattern. With increasing $N$, as seen in Fig. 4(d), the shallow grooves grow up to form a nanograting (thin white arrows) with $d \sim \Lambda$ / $4=350 \mathrm{~nm}$.

These results demonstrate that the nanograting formation is dominated by the ablation due to the periodically enhanced near-fields of SPPs ${ }^{32}$ with a uniform peak intensity. In addition, the grating period $d \sim \Lambda / q$ given by a single integer $q$ ensures the excitation of a single spatial standing SPP wave mode, as discussed in detail in our recent study. ${ }^{17}$ The SPP waves propagating in opposite directions on the fringe can induce the line-like ablation even at the midway of two adjacent fringes.

For simulating the excitation of SPPs with a nanoperiod, we note that PNSs have been observed so far only for such metals that thin passive oxide layers are spontaneously formed on the surface in air. ${ }^{7,8,18-28}$ For the SUS and Ti concerned, the surfaces are covered mainly with a thin layer of $\mathrm{Cr}_{2} \mathrm{O}_{3}{ }^{33}$ and $\mathrm{TiO}_{2}{ }^{23}$ respectively. The native oxide layer is a few to several nanometers in thickness, which would be further developed in air with the local-temperature increase due to irradiation of intense fs laser pulses. Then, modeling the initial target surface as schematically shown in Fig. 5(a), we consider the growth of surface corrugation and resulting excitation of SPPs at the interface between the dielectric oxide layer (layer $o x$ ) and the metal substrate (layer $s$ ), based on the experimental results.

As the oxide layer of a fresh surface is almost transparent at $\lambda \sim 800 \mathrm{~nm}$, the incident laser energy is initially absorbed at the skin depth in the metal substrate. Even at a low fluence concerned, the field intensity at the interference fringe is high enough to give rise to the emission of photoelectrons from the substrate into the oxide layer. ${ }^{34}$ Energy transfer from the emitted electrons to the lattice would modify the bonding structure in the oxide layer, e.g., to increase the defect density. The accumulated bonding structure change inevitably induces small surface swelling, being (a)

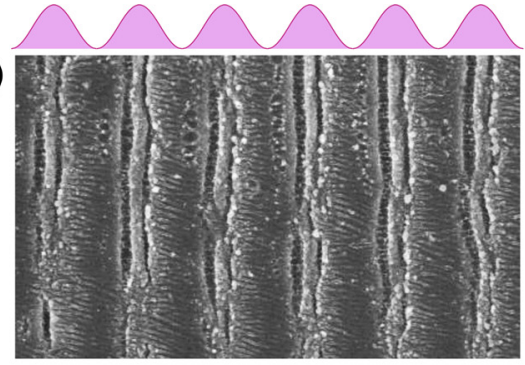



(b)

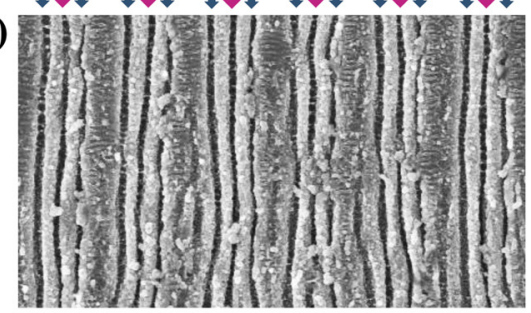



(c)

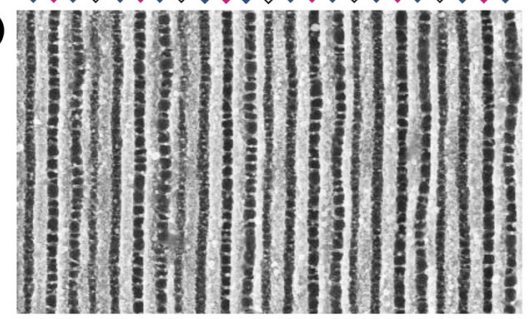

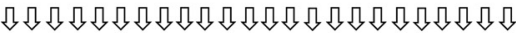

(d)

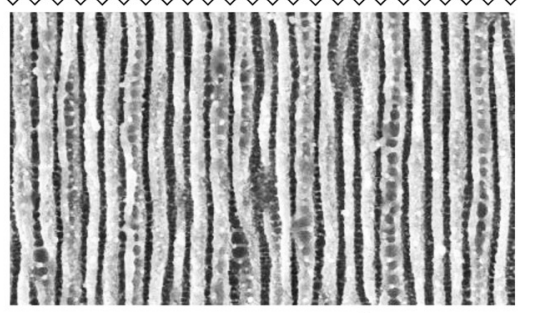

FIG. 4. SEM images of SUS surface irradiated with (a) $N=150$, (b) $N=250$ at $F=50 \mathrm{~mJ} / \mathrm{cm}^{2}$, and with (c) $N=50$, (d) $N=100$ at $F=100 \mathrm{~mJ} /$ $\mathrm{cm}^{2}$ for $\Lambda=1400 \mathrm{~nm}$, where the laser polarization is horizontal. For reference, (a) includes the scheme of a modulated intensity distribution in the interference; in (b) and (c), a set of three line-like grooves on the fringe is indicated by a thick solid arrow at the peak intensity and two narrow solid arrows on its both sides; in (d), the white arrows represent the groove positions almost regulated at a period $d \sim \Lambda / 4$. 
often accompanied with random ablation on the nanoscale. ${ }^{13,15}$ This initial interaction can be seen with the bright fringe pattern in the SEM images, e.g., in Figs. 2(a) and 4(a). An increase in $N$ would induce ionization of material at the fringe and resulting polarization-dependent plasmonic nearfields to form the narrow line-like grooves, as seen in Fig. 4(a). The near-field ablation rate tends to decrease soon due to the small field size, ${ }^{35}$ while the initial grooves create new high curvatures to induce other near-fields. The successive near-field ablation creates multiple line-like grooves on the fringe, as seen in Figs. 4(b) and 4(c). The periodic grooves on the fringe coherently couple the incident laser field with SPPs.

The method of calculating the SPP wavelength $\lambda_{\text {spp }}$ on the corrugated surface is almost the same as those in our previous studies ${ }^{15-17}$ using the relation for the SPP wave number

$$
k_{\mathrm{spp}}=k_{0}\left[\varepsilon_{\mathrm{s}} \varepsilon^{*} /\left(\varepsilon_{\mathrm{s}}+\varepsilon^{*}\right)\right]^{1 / 2},
$$

where $k_{0}$ is the wave number of incident light in vacuum and $\varepsilon^{*}$ and $\varepsilon_{\mathrm{s}}$ are the relative dielectric constants of the excited oxide layer and the metal substrate, respectively. As the oxide layer is ionized by the fs laser pulse, $\varepsilon^{*}$ would rapidly be changed as $\varepsilon^{*}=\varepsilon_{\mathrm{ox}}-\left[\omega_{\mathrm{p}}{ }^{2} /\left(\omega^{2}+i \omega / \tau\right)\right]$ during the interaction. Here, $\varepsilon_{\mathrm{ox}}$ is the dielectric constant of the original oxide layer, and the second term represents the effect of the free electron density $N_{\mathrm{e}}$ produced in the oxide layer, where $\omega$ is the laser frequency in vacuum, $\tau$ is the Drude damping time of free electrons, and $\omega_{\mathrm{p}}=\left[e^{2} N_{\mathrm{e}} /\left(\varepsilon_{0} m^{*} m\right)\right]^{1 / 2}$ is the plasma frequency with the dielectric constant of vacuum $\varepsilon_{0}$, the electron charge $e$ and mass $m$, and the optical effective mass of electrons $m^{*}$. In the calculation, we used $\varepsilon_{\mathrm{s}}=-13.4+i 29.8$ for $\mathrm{SUS},{ }^{36} \varepsilon_{\mathrm{S}}=-2.85+i 19.1$ for $\mathrm{Ti}^{37}{ }^{37} \varepsilon_{\mathrm{ox}}=4.84+i 0.220$ for $\mathrm{Cr}_{2} \mathrm{O}_{3},{ }^{36}$ and $\varepsilon_{\mathrm{ox}}=7.78+i 0.446$ for $\mathrm{TiO}_{2},{ }^{37}$ and assumed $m^{*}=0.8$ (Ref. 38) and $\tau=1$ fs (Ref. 39) for both oxides.

Figure 5(b) shows the nanograting period $D=\lambda_{\text {spp }} / 2=\pi /$ $\left(\operatorname{Re}\left[k_{\text {spp }}\right]\right)$ calculated as a function of $N_{\mathrm{e}}$. The excitation of SPPs at the interface between the layers $o x$ and $s$ is allowed for $\operatorname{Re}\left[\varepsilon^{*}\right] \times \operatorname{Re}\left[\varepsilon_{\mathrm{s}}\right]<0,{ }^{32}$ which corresponds to the region of $N_{\mathrm{e}}<7.9 \times 10^{21} \mathrm{~cm}^{-3}$ for SUS and $N_{\mathrm{e}}<1.3 \times 10^{22} \mathrm{~cm}^{-3}$ for Ti. The results suggest that $D$ should be in a range of $190 \mathrm{~nm}<D<390 \mathrm{~nm}$ for SUS and $160 \mathrm{~nm}<D<330 \mathrm{~nm}$ for Ti, where the lower values are taken at $N_{\mathrm{e}} \sim 1 \times 10^{21} \mathrm{~cm}^{-3}$ as a minimum value of $N_{\mathrm{e}}$ for ablation. ${ }^{10,39}$ It is noted that $D$ is consistent with the observed period $d \sim \Lambda / 3=295 \mathrm{~nm}$ with $\Lambda=890 \mathrm{~nm}$ for SUS and Ti [Figs. 2 and 3] and $d \sim \Lambda$ / $4=350 \mathrm{~nm}$ with $\Lambda=1400 \mathrm{~nm}$ for SUS [Fig. 4].

Using $\varepsilon^{*}$, we calculated the skin depth $\delta$ in the excited oxide layers as a function of $N_{\mathrm{e}}$. The results have shown that $\delta \sim 500 \mathrm{~nm}$ at $N_{\mathrm{e}} \sim 10^{21} \mathrm{~cm}^{-3}$ rapidly decreases to $\delta \sim 60 \mathrm{~nm}$ in $\mathrm{Cr}_{2} \mathrm{O}_{3}$ and to $\delta \sim 45 \mathrm{~nm}$ in $\mathrm{TiO}_{2}$ at $\operatorname{Re}\left[\varepsilon^{*}\right]=0$. Meanwhile, we have $\delta \sim 13 \mathrm{~nm}$ and $\sim 19 \mathrm{~nm}$ for SUS and Ti substrates, respectively. In the present experiment, the ablation rate measured with the SPM was $10 \mathrm{~nm}$ per pulse at most. These values justify the interaction that the incident laser pulse passes through the excited oxide layer of $\sim 10 \mathrm{~nm}$ or less in thickness and penetrates into the substrate to excite SPPs at the interface between the layers $o x$ and $s$. Once ablation takes (a)

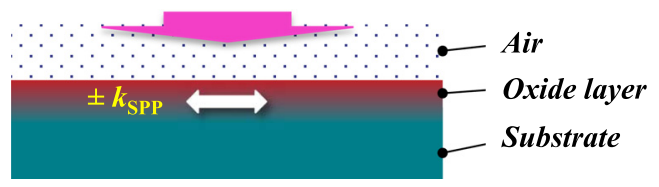

(b)

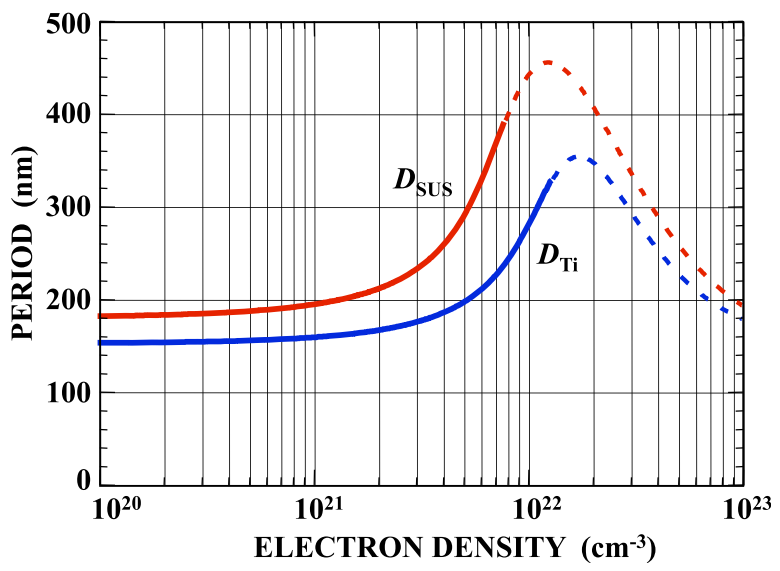

FIG. 5. (a) Schematic of the initial target surface modeled for calculation. SPPs (left right arrow) are excited at the interface between the oxide layer and the metal substrate, when the surface is corrugated with multiple laser pulses (thick down arrow). (b) Calculated groove periods $D_{\text {SUS }}$ for SUS (upper curve) and $D_{\mathrm{Ti}}$ for Ti (lower curve) as a function of $N_{\mathrm{e}}$ in the oxide layer, where the excitation of SPPs is allowed in the region (solid curve) of $0<\operatorname{Re}\left[\varepsilon^{*}\right]$.

place, the metal substrate rapidly reacts with $\mathrm{O}_{2}$ from air to form the oxide layer.

In conclusion, using interfering fs-laser pulses, we have demonstrated that a homogeneous nanograting can be fabricated on SUS and Ti surfaces, through the ablation induced by SPPs in a single spatial standing wave mode. The calculation for a model target has reproduced well the observed groove period to illustrate the interaction mechanism.

This work was partially supported by the Grant-in-Aid for Scientific Research 23360034, 24686011, and 22110709.

${ }^{1}$ J. Bonse, H. Sturm, D. Schmidt, and W. Kautek, Appl. Phys. A: Mater. Sci. Process. 71, 657 (2000).

${ }^{2}$ J. Reif, F. Costache, M. Henyk, and S. V. Pandelov, Appl. Surf. Sci. 197-198, 891 (2002).

${ }^{3}$ N. Yasumaru, K. Miyazaki, and J. Kiuchi, Appl. Phys. A: Mater. Sci. Process. 76, 983 (2003).

${ }^{4}$ Q. Wu, Y. Ma, R. Fang, Y. Liao, Q. Yu, X. Chen, and K. Wang, Appl. Phys. Lett. 82, 1703 (2003).

${ }^{5}$ A. Borowiec and H. K. Haugen, Appl. Phys. Lett. 82, 4462 (2003).

${ }^{6}$ G. Daminelli, J. Krüger, and W. Kautek, Thin Solid Films 467, 334 (2004).

${ }^{7}$ J. Bonse, J. Krüger, S. Höhm, and A. Rosenfeld, J. Laser Appl. 24, 042006 (2012), and references therein.

${ }^{8}$ K. Miyazaki and G. Miyaji, Appl. Phys. A: Mater. Sci. Process. 114, 177 (2014), and references therein.

${ }^{9}$ K. Miyazaki and G. Miyaji, Phys. Precedia 39, 674 (2012).

${ }^{10}$ G. Miyaji and K. Miyazaki, Appl. Phys. Lett. 103, 071910 (2013).

${ }^{11}$ N. Yasumaru, K. Miyazkai, and J. Kiuchi, Appl. Phys. A: Mater. Sci. Process. 79, 425 (2004).

${ }^{12}$ K. Miyazaki, N. Maekawa, W. Kobayashi, M. Kaku, N. Yasumaru, and J. Kiuchi, Appl. Phys. A: Mater. Sci. Process. 80, 17 (2005).

${ }^{13}$ G. Miyaji and K. Miyazaki, Appl. Phys. Lett. 89, 191902 (2006).

${ }^{14}$ G. Miyaji and K. Miyazaki, Appl. Phys. Lett. 91, 123102 (2007).

${ }^{15}$ G. Miyaji and K. Miyazaki, Opt. Express 16, 16265 (2008).

${ }^{16}$ G. Miyaji, K. Miyazaki, K. Zhang, T. Yoshifuji, and J. Fujita, Opt. Express 20, 14848 (2012). 
${ }^{17}$ K. Miyazaki and G. Miyaji, J. Appl. Phys. 114, 153108 (2013).

${ }^{18}$ L. Qi, K. Nishii, and Y. Namba, Opt. Lett. 34, 1846 (2009).

${ }^{19}$ S. Hou, Y. Huo, P. Xiong, Y. Zhang, S. Zhang, T. Jia, Z. Sun, J. Qiu, and Z. Xu, J. Phys. D: Appl. Phys. 44, 505401 (2011).

${ }^{20}$ N. Yasumaru, K. Miyazaki, and J. Kiuchi, Appl. Surf. Sci. 264, 611 (2013).

${ }^{21}$ E. V. Golosov, V. I. Emel'yanov, A. A. Ionin, Yu. R. Kolobov, S. I. Kudryashov, A. E. Ligachev, Yu. N. Novoselov, L. V. Seleznev, and D. V. Sinitsyn, JETP Lett. 90, 107 (2009).

${ }^{22}$ C. Wang, H. Huo, M. Johnson, M. Shen, and E. Mazur, Nanotechnology 21, 075304 (2010).

${ }^{23}$ J. Bonse, S. Höhm, A. Rosenfeld, and J. Krüger, Appl. Phys. A: Mater. Sci. Process. 110, 547 (2013)

${ }^{24}$ X. Li, C. Zhang, H. Li, Q. Dai, S. Lan, and S. Tie, Opt. Express 22, 28086 (2014).

${ }^{25}$ A. Weck, T. H. R. Crawford, D. S. Wilkinson, H. K. Haugen, and J. S. Preston, Appl. Phys. A: Mater. Sci. Process. 89, 1001 (2007).

${ }^{26}$ K. Okamuro, M. Hashida, Y. Miyasaka, Y. Ikuta, S. Tokita, and S. Sakabe, Phys. Rev. B 82, 165417 (2010).

${ }^{27}$ A. A. Ionin, S. I. Kudryashov, S. V. Makarov, A. A. Rudenko, P. N. Saltuganov, L. V. Seleznev, D. V. Sinitsyn, and E. S. Sunchugasheva, Appl. Surf. Sci. 292, 678 (2014).
${ }^{28}$ C. Albu, A. Dinescu, M. Filipescu, M. Ulmeanu, and M. Zamfirescu, Appl. Surf. Sci. 278, 347 (2013).

${ }^{29}$ J. E. Sipe, J. F. Young, J. S. Preston, and H. M. van Driel, Phys. Rev. B 27, 1141 (1983).

${ }^{30}$ See, e.g., B. E. A. Saleh and M. C. Teich, Fundamentals of Photonics (John Wiley \& Sons, New York, 1991), Chap. 2.5

${ }^{31}$ G. Obara, N. Maeda, T. Miyanishi, M. Terakawa, N. N. Nedyalkov, and M. Obara, Opt. Express 19, 19093 (2011).

${ }^{32}$ H. Raether, Surface Plasmons on Smooth and Rough Surfaces and on Gratings (Springer, Berlin, 1988).

${ }^{33}$ K. Nomura and Y. Ujihara, J. Mater. Sci. 25, 1745 (1990).

${ }^{34}$ Z. Chen and S. S. Mao, Appl. Phys. Lett. 93, 051506 (2008).

${ }^{35}$ J. B. Nielsen, J. M. Savolainen, M. S. Christensen, and P. Balling, Appl. Phys. A: Mater. Sci. Process. 101, 97 (2010).

${ }^{36}$ B. Karlsson and C. G. Ribbing, J. Appl. Phys. 53, 6340 (1982).

${ }^{37}$ E. D. Palik, Handbook of Optical Constants of Solids (Academic, London, 1998).

${ }^{38}$ T. V. Perevalov and V. A. Gritsenko, J. Exp. Theor. Phys. 112, 310 (2011).

${ }^{39}$ K. Sokolowski-Tinten and D. von der Linde, Phys. Rev. B 61, 2643 (2000). 